\title{
Cold Atmospheric Plasma (CAP) in Dentistry
}

\section{Arora $\mathbf{V}^{1 *}$, Nikhil $\mathbf{V}^{1}$, Suri $\mathrm{NK}^{1}$ and Arora $\mathrm{P}^{2}$}

${ }^{1}$ Department of Conservative Dentistry and Endodontics, Subharti Dental College \& Hospital, India

${ }^{2}$ Department of Prosthodontics and Dental Materials, Subharti Dental College \& Hospital, India

\begin{abstract}
This review article on plasma therapy in dentistry is intended to provide with a summary of the current status of this emerging field, its scope, and its broad interdisciplinary approach. Matter usually includes liquids, solids, and gases. But a fourth category of matter has been discovered called plasma that's actually the most unusual and the most abundant. It could become a new and painless way to prepare cavities for restoration with improved longevity. Also it is capable of bacterial inactivation and non-inflammatory tissue modification, which makes it an attractive tool for the treatment of dental caries and for composite restorations. Plasma can also be used for tooth whitening. This review focuses on some dental applications of plasma.
\end{abstract}

Keywords: Cold Atmospheric Plasma (CAP); Dental caries; Plasma; Dentin/adhesive interfacial bonding; Sterilization; Tooth whitening

\section{Introduction}

Plasma is the fourth state of matter, discovered by the British physicist Sir William Crookes in the year 1879, but the name "plasma" was applied by Irving Langmuir, an American chemist, in 1929. As the most common form of matter, it makes up for more than 99 percent of the visible universe; plasma is a collection of stripped particles. Once the electrons are stripped from atoms and molecules, those particles change state and become plasma. Plasmas are naturally energetic because stripping electrons uses constant energy. If the energy dissipates, the electrons reattach and the plasma particles become a gas once again. Unlike ordinary matter, plasmas can exist in a wide range of temperatures without changing state. The aurora borealis, or northern lights, is ice cool, for instance, while the core of a distant star is white hot. Other well-known plasmas include lightning, neon signs, and fluorescent lights. Outside of a container, plasma resembles gasthe particles don't have a definite shape. But unlike gas, magnetic and electric fields can control plasma and shape it into useful, malleable structures [1]

Based on the relative temperatures of the electrons, ions and neutrals, plasmas are classified as "thermal" or "non-thermal". Thermal plasmas have electrons and the heavy particles at the same temperature, i.e., they are in thermal equilibrium with each other. Non-thermal plasmas on the other hand have the ions and neutrals at a much lower temperature (sometimes room temperature), whereas electrons are much "hotter". In recent years, cold (less than $40{ }^{\circ} \mathrm{C}$ at the point of application) atmospheric plasma (CAP) sources have been introduced that provide the possibility to extend plasma treatment to living tissue [2].

Cold Atmospheric Plasma (CAP) is known as non-thermal because it has electrons at a hotter temperature than the heavy particles that are at room temperature. Its temperature is less than $104^{\circ} \mathrm{F}$ at the point of application. Various methods to produce include Dielectric Barrier Discharge (DBD), Atmospheric Pressure Plasma Jet (APPJ), plasma needle, and plasma pencil. Gases that can be used to produce CAP are Helium, Argon, Nitrogen, Heliox (a mix of helium and oxygen), and air. Due to its ability to deactivate microorganisms, cause cell detachment, and cause death in cancer cells, researchers have been interested in finding uses for CAP in dentistry and oncology [3].

Applications of plasma for sterilization of medical equipment, packaging in the food industry, implants, wound healing; blood coagulation, etc have been known [4-11]. This has been possible due to their high bactericidal effectiveness and partly due to their easy access into narrow and confined spaces [12-17]. As conventional methods have numerous drawbacks, low-temperature plasma can be used as an alternative method for destroying microorganisms [12].

Dental applications of CAP include: dental caries, sterilization, elimination of biofilms, root canal disinfection, increase in bond strength at the dentin/composite interface and bleaching. Various means of producing plasmas include: radio frequency, microwave frequencies, high voltage $\mathrm{AC}$ or $\mathrm{DC}$ etc. This review is an update about the novel technique using plasma in dentistry.

\section{Nonthermal Plasma Characteristics and Sources}

One of the important features of non-thermal plasma is the abundant production of reactive species in low gas temperature which includes charged particles, radiation, and reactive oxygen species. The energy for sustaining the plasma state is usually supplied by electromagnetic field. Electrons are accelerated by the field much faster, but are less effective to transfer their energies to heat their environment than heavy ions. The plasma can remain non-thermal, the energetic electrons can lead to reactions including ionization of particles, production of reactive species, and radiation [18].

The complex components from non-thermal plasmas achieve multi-functional treatment in oral cavity. For example, reactive oxygen species and reactive nitrogen species are regarded as a key factor for sterilization, wound healing, and tooth whitening. Concentration of the components can be controlled by the plasma operating conditions, making it possible that non-thermal plasmas be employed for various biomedical applications.

Various plasma sources have been developed to treat the human body; these include dielectric barrier discharges (DBD)-type surface plasma devices, and pulsed power sources [19]. In these devices,

${ }^{*}$ Corresponding author: Vipin Arora, Professor and HOD, Department of Conservative Dentistry and Endodontics, Subharti Dental College and Hospital, India, Tel: 91980816252; E-mail: vipin_endodontist@yahoo.co.in

Received December 03, 2013; Accepted December 31, 2013; Published January 02, 2014

Citation: Arora V, Nikhil V, Suri NK, Arora P (2014) Cold Atmospheric Plasma (CAP) in Dentistry. Dentistry 4: 189. doi:10.4172/2161-1122.1000189

Copyright: ( 2014 Arora V, et al. This is an open-access article distributed under the terms of the Creative Commons Attribution License, which permits unrestricted use, distribution, and reproduction in any medium, provided the original author and source are credited. 
electrical safety is achieved by limiting the current flow. The DBD configuration prevents current-to-transit arcing. Use of a pulsed signal also limits the current due to its off time. Microwave-driven plasma is stable and safe, and hence, it is a promising candidate for biomedical applications [20,21].

\section{Plasma in Dentistry}

Oral infections, including dental caries, periodontal disease, and intraoral disease, are caused by bacteria and may result in tooth destruction [22]. Although teeth brushing, fluoride uptake, antibiotics, and vaccines have been used as treatment modalities for oral disease, these conventional treatments have limitations [23].

Heat kills bacteria, but the application of this method to living tissues is dangerous. Sterilizing agents or antibiotics are used to treat human tissues that are infected by pathogens, but this may lead to pain and antibiotic resistance. Recently, non-thermal atmospheric plasmas have been shown to be highly efficient at killing bacteria in an inexpensive manner; therefore, the use of such plasmas could eliminate the problems associated with use of heat and antibiotics.

\section{The Application of Non-thermal Plasmas/CAP to Dental Diseases}

\section{Sterilization by eradication of bacteria}

The sterilization efficacy of plasma devices is influenced by gas composition, driving frequency, and bacterial strain, but plasma devices have shown to kill a higher proportion of bacteria than do conventional non-thermal methods such as UV sterilization [24,25]. The mechanism of plasma sterilization is related to the abundance of plasma components, like reactive oxygen species, ions and electrons, and UV and electromagnetic fields [26]. Also, plasma can affect not only the contacted point but also the area around it. Recently, plasma sterilization has been used to treat dental diseases [6].

The risk of prior transmission through surgical instruments is of both current public and professional concern. The use of plasma decontamination of surgical instruments is limited. Whittaker et al. has indicated that the use of gas plasma cleaning may be extremely beneficial in reducing the absolute amount of proteinaceous materials that may be transferred between patients when endodontic files are reused [27].

Yang Hong Li et al. stated that plasma sterilization, with the advantage of low temperature, fastness, thoroughness, safety, overcomes the deficiency of the traditional sterilization technology, and may become a novel method for killing microbe [28].

Autoclaves and UV sterilizers are presently used to sterilize dental instruments. To develop a dental sterilizer which can sterilize most materials, such as metals, rubbers, and plastics, the sterilization effect of an atmospheric pressure non-thermal air plasma device was evaluated by $\mathrm{Su}$-Jin Sung et al. It was proved that the atmospheric pressure nonthermal air plasma device was effective in killing both Escherichia coli and Bacillus subtilis, and was more effective in killing Escherichia coli than the UV sterilizer [29].

\section{Dental caries}

Plasmas can treat and sterilize irregular surfaces; making them suitable for decontaminating dental cavities without drilling. Although plasma itself is superficial, the active plasma species it produces can easily reach inside of the cavity. This approach was pioneered by Eva
Stoffels, who suggested the use of plasma needles in the dental cavity on the basis of the ability of plasma to kill Escherichia coli [30]. Goree et al. provided substantial evidence that non thermal atmospheric plasmas killed Streptococcus mutans, a gram-positive cariogenic bacterium [31]. Sladek et al. studied the interactions of the plasma with dental tissue using a plasma needle [30]. It is an efficient source of various radicals, which are capable of bacterial decontamination; but, it operates at room temperature and thus, does not cause bulk destruction of the tissue. From his research he concluded that plasma treatment is potentially a novel tissue-saving technique, which allows irregular structures and narrow channels within the diseased tooth to be cleaned.

Subsequently, a low-temperature atmospheric argon plasma brush introduced by Yang et al. was found to be very effective in deactivating Streptococcus mutans and Lactobacillus acidophilus [32]. These authors concluded that about $100 \%$ bacterial elimination was achieved within $15 \mathrm{~s}$ for Streptococcus mutans and in $5 \mathrm{~min}$ for Lactobacillus acidophilus. In comparison to lasers, plasmas can access small irregular cavities and fissure spaces.

\section{Biofilms}

Biofilms develop on tooth and oral mucosa, cause caries, periodontal diseases, and oral mucositis, which can also lead to inflammation around dental implants. Rupf et al. demonstrated that combination treatment with plasma and a non-abrasive air/water spray is suitable for the elimination of oral biofilms from microstructured titanium used in dental implants [33]. Furthermore, Koban et al. showed that the treatment of dental biofilms composed of Streptococcus mutans with non-thermal plasma was more efficient than the treatment with chlorhexidine in vitro [34].

CAP was also effective in destroying biofilms either on root canals or on dental slices. Jiang et al. developed a plasma plume at room temperature. They used it to disinfect root canals from extracted human teeth [35]. Two teeth were placed at a distance of $5 \mathrm{~mm}$ from the plasma nozzle. One of them was exposed to the helium/oxygen plasma for 5 minutes, whereas the other one was exposed to the same helium/ oxygen flow for five minutes, but without plasma. They observed better results in the reduction of the biofilms in the tooth treated with plasma compared with control. Nevertheless, the plasma failed to reach the lower zone of the tooth. The authors explained it by the fact that the plasma plume did not have the optimal width and length to effectively treat the lower zone.

Schaudinn et al. used a plasma needle to eliminate ex vivo biofilms on root canals of extracted teeth [36].Teeth were divided into three groups: treatment with the plasma needle, treatment with $6 \%$ sodium hypochlorite (an antiseptic), and control. It was concluded that the plasma needle was effective at killing biofilms in extracted teeth. But, using $6 \%$ sodium hypochlorite was more efficient.

\section{Intraoral diseases}

Oral candidiasis includes Candida-associated denture stomatitis, angular stomatitis, median rhomboid glossitis, and linear gingival erythema. Koban et al. and Yamazaki et al. reported the high efficiency of Candida albicans sterilization using various plasmas. Their result indicates the possibility that stomatitis caused by Candida albicans can be cured by plasma jets $[37,38]$.

\section{Root canal disinfection}

Treatment of root canal infection (periapical abscess) is difficult. Because it is difficult to penetrate irregular and narrow spaces, killing 
the pathogens; thus, infections frequently recur. Lu et al. used a reliable and user-friendly plasma-jet device that could generate plasma inside the root canal [39]. Preliminary inactivation experiment results showed that it can efficiently kill Enterococcus faecalis one of the main types of bacterium causing failure of root-canal treatment in several minutes. Thus, plasma can be utilized in disinfection of root canals.

Pan et al. investigated the feasibility of using a cold plasma treatment of a root canal infected with Enterococcus faecalis biofilms in vitro [40]. It was concluded that the cold plasma had a high efficiency in disinfecting the Enterococcus faecalis biofilms in vitro dental root canal treatment.

\section{Disinfection of dental surfaces}

In a new report, Rupf et al. used atmospheric plasma jets in dental caries causing organisms [41]. The objective of their in vitro study was to test a microwave-powered non-thermal atmospheric plasma jet for its antimicrobial efficacy against adherent oral micro-organisms. The plasma-jet treatment reduced the CFU by 3-4 $\log 10$ intervals on the dentin slices in comparison to the recovery rates from untreated controls. Thus, non-thermal atmospheric plasma jets could also be used for the disinfection of dental surfaces.

\section{Adhesive restorations}

Preliminary data has shown that plasma treatment increases bonding strength at the dentin/ composite interface by roughly 60 percent, and with that interface-bonding enhancement to significantly improve composite performance, durability, and longevity.

Kong et al. investigated the plasma treatment effects on dental composite restoration for improved interface properties and their results showed that atmospheric cold plasma brush (ACPB) treatment can modify the dentin surface and thus increase the dentin/adhesive interfacial bonding. The solution is to introduce bonds that depend on surface chemistry rather than surface porosity [42].

Ritts et al. investigated a non-thermal atmospheric plasma brush on dental composite restoration [43]. It was observed that atmospheric cold plasma brush (ACPB) treatment could modify the dentin surface and increase dentin/adhesive interfacial bonding. Yavirach et al. studied the effects of plasma treatment on the shear bond strength between fiber reinforced composite posts and resin composite for core buildup and concluded that plasma treatment increased the tensile-shear bond strength between post and composite [44].

\section{Tooth whitening}

CAP can also be used to bleach teeth. Lee et al. showed that atmospheric pressure plasma in place of light sources bleached teeth by increasing the production of $\mathrm{OH}$ radicals and the removal of surface proteins [45]. Furthermore, Lee et al. also showed that in combination with hydrogen peroxide, this plasma removed stains from extracted teeth stained by either coffee or wine [46]. The tooth bleaching method using an atmospheric pressure jet shows reasonable promise of becoming practical in the future. Tooth whitening can also be achieved using a DC plasma jet and hydrogen peroxide [47].

Intrinsic stains are a serious factor in tooth discoloration. Park et al. suggested intrinsic whitening using a low-frequency plasma source and hydrogen peroxide [48]. Another approach, by Kim et al. used liquid plasma produced by an RF driven gas-liquid hybrid plasma system [49]. In this study, the RF plasma jet was placed in deionized water and the target tooth was immersed in the water. Color changes were observed on the surface of the treated tooth after $8 \mathrm{~min}$. The $\mathrm{OH}$ radicals were regarded as the main cause of bleaching in this work also.

Nam et al. used a Plasma jet on forty extracted human molar teeth with intact crowns. The forty teeth were randomly divided into four groups $(\mathrm{n}=10)$ and were treated with Carbamide peroxide + CAP, Carbamide peroxide + Plasma Arc Lamp (PAC), Carbamide peroxide + diode laser, or Carbamide Peroxide alone (control). They observed CAP was the most effective at bleaching teeth. Moreover, they observed that CAP does not damage the tooth due to its low temperature [50].

Claiborne D et al. used a plasma plume on extracted human teeth. They observed a statistically significant increase in the whitening of the teeth after exposure to CAP $+36 \%$ hydrogen peroxide gel, compared with $36 \%$ hydrogen peroxide only, in the 10 and $20 \mathrm{~min}$ groups. The temperature in both treatment groups remained under $80^{\circ} \mathrm{F}$ throughout the study, which is below the thermal threat for vital tooth bleaching [51].

In a study by Jamali et al. results revealed that prolonged plasma treatment (without bleaching) removed some blue-stain, but the effect was small [52]. On the contrary, the combination of plasma treatment and bleaching removed most of the blue-stain. It was concluded that vacuum plasma pre-treatment and bleaching showed promise as a way of removing blue-stain.

Nam et al. investigated the efficacy of tooth bleaching using nonthermal atmospheric pressure plasma (NAPP) with 15\% carbamide peroxide including 5.4\% hydrogen peroxide, as compared with conventional light sources. It was observed that the NAPP has a greater capability for effective tooth bleaching than conventional light sources with a low concentration of hydrogen peroxide without causing thermal damage [50].

Application of plasma treatment in dental treatment procedures may effectively disinfect cavity-causing bacteria; reduce the use of the painful and destructive drilling, and consequently save healthy dental tissues. Not only contact free sterilization be obtained, accessibility of even small pores and microscopic openings, but also one may envisage new possibilities of drug delivery at the molecular level in the dental tissues.

\section{Discussion}

Plasma is a gaseous medium which can penetrate into irregular cavities and fissures. In comparison to laser beams, which propagate linearly, plasma has many useful advantages in its application to oral tissues. Moreover, plasma has the strong advantage in that it kills only pathogens in bacterial plaque on oral tissues, without damaging the normal tissue. Plasma does not cause pain in patients, because it does not induce thermal damage. Since, oral diseases are not caused by a single pathogen; research must be conducted as to whether non thermal atmospheric plasmas can also kill various other oral pathogens at the same time. Considering all the characteristics of plasmas, such as its sterilizing effect, blood coagulation, wound healing, and tooth bleaching, the application of plasma to oral tissues is potentially a fascinating novel technique in dental care.

Based on known physical and biological properties of plasma, it is worthwhile to speculate that a number of dental applications are possible, but fundamental principles of how plasma influences cells and effect time needs further investigation. Earlier, Plasma Dent was just speculation, but plasma-assisted dental devices will unquestionably be available in the future to augment or replace existing technologies and therapies-the untapped potential of plasma in dentistry is limitless. Although it is not possible to include all the aspects of plasma therapy in 
this article, we are still far away from an implacable dental and medical plasma application; it is just intent to create an awareness towards safe, developing, efficient and echo- friendly plasma technology [53].

\section{Limitations}

As we all know that every technology has its own advantages and limitations.CAP also has some limitations as this is a new technology, safety of the equipment has to be taken care of [7,9]. Cost of the equipment, marketing, maintenance and availability are also some of the issues at present [1]. Portability of instrument for dental use is also one factor [22]. Now-a-days, research of the effect of CAP on tumor cells is being done and some promising results are there, but the effect on normal cells has to be studied in depth and validation needed for its successful application [54-56]. This is a beginning and still some research is needed for this technology to be used in a cost effective, efficient and predictable manner in clinical settings.

\section{Conclusion}

Based on the above evidence, we can say that CAP has a bright future in dentistry due to its anti-microbial properties and its cell death properties on cells. It can be used in almost all the branches of dentistry. However, more studies need to be performed regarding the mechanism of action.

\section{References}

1. Martin M (2009) From distant stars to dental chairs- Plasmas May Promise Pain-free and durable Restorations. AGD Impact 37: 46.

2. Kong MG, Kroesen G, Morfill G, Nosenko T, Shimizu T, et al. (2009) Plasma medicine: an introductory review. New J Phys 11: 115012

3. Hoffmann C, Berganza C, Zhang J (2013) Cold Atmospheric Plasma: methods of production and application in dentistry and oncology. Med Gas Res 3: 21.

4. Deng S, Ruan R, Mok CK, Huang G, Lin X, et al. (2007) Inactivation of Escherichia coli on almonds using non thermal plasma. J Food Sci 72: 62-66.

5. Deilmann M, Halfmann H, Bibinov N, Wunderlich J, Awakowicz P (2008) Lowpressure microwave plasma sterilization of polyethylene terephthalate bottles. J Food Prot 71: 2119-2123.

6. Fridman G, Fridman G, Gutsol A, Shekhter AB, Vasilets VN, Fridman A (2008) Applied plasma medicine. Plasma Process Polym 5: 503-533.

7. Moreau M, Orange N, Feuilloley MGJ (2008) Non-thermal plasma technologies: new tools for bio-decontamination. Biotechnol Adv 26: 610-617.

8. Selcuk M, Oksuz L, Basaran P (2008) Decontamination of grains and legumes infected with Aspergillus spp. and Penicillum spp. by cold plasma treatment. Bioresour Technol 99: 5104-5109.

9. Deng X, Shi JJ, Kong MG (2007) Protein destruction by a helium atmospheric pressure glow discharge: capability and mechanisms. J Appl Phys 101: 074701.

10. Morrison CF (1977) Electrosurgical method and apparatus for initiating an electrical discharge in an inert gas flow.US Patent No. 4,040,426.

11. Farin G, Grund KE (1994) Technology of argon plasma coagulation with particular regard to endoscopic applications. Endosc Surg Allied Technol 2 : 71-77.

12. Lerouge S, Wertheimer MR, Yahia LH (2001) Plasma sterilization: a review of parameters, mechanisms, and limitations. Plasmas Polym 6: 175-188.

13. Moisan M, Barbeau J, Moreau S, Pelletier J, Tabrizian M, et al. (2001) Lowtemperature sterilization using gas plasmas: a review of the experiments and an analysis of the inactivation mechanisms. Int J Pharm 226: 1-21.

14. Laroussi M (2002) Non-thermal decontamination of biological media by atmospheric pressure plasmas: review, analysis and prospects IEEE Trans. Plasma Sci 30: 1409-1415.

15. Sharma A, Pruden A, Zengqi Y, Collins GJ (2005) Bacterial inactivation in open air by the afterglow plume emitted from a grounded hollow slot electrode. Environ Sci Technol 39: 339-344.
16. Sladek RE, Stoffels E (2005) Deactivation of Escherichia coli by the plasma needle. J Phys D: Appl Phys 38: 1716-1721.

17. Laroussi M, Mendis DA, Rosenberg M (2003) Plasma interactions with microbes. New J Phys 5: 1-41.

18. Iza F, Kim GJ, Lee SM, Lee JK, Walsh JL, et al. (2008) Microplasmas: Sources, Particle Kinetics and Biomedical Applications. Plasma Process Polym 5: 322344

19. Laroussi M, Akan T (2007) Arc-free Atmospheric Pressure Cold Plasma Jets: A Review. Plasma Process Polym 4: 777-788.

20. Choi J, Iza F, Do HJ, Lee JK, Cho MH (2009) Microwave-excited atmosphericpressure microplasmas based on a coaxial transmission line resonator. Plasma Sources Sci Technol 18: 025029.

21. Park SJ, Choi J, Park GY, Lee SK, Cho Y, et al. (2010) Inactivation of S. Mutans Using an Atmospheric Plasma Driven by a Palm-Size-Integrated MicrowavePower Module. IEEE Trans Plasma 38: 1956-1962.

22. Khalil J (2008) Periodontal disease: an overview for medical practitioners. Lik Sprava 3: 10-21.

23. Tenuta LM, Zamataro CB, Del BelCury AA, Tabchoury CP, Cury JA (2009) Mechanism of fluoride dentifrice effect on enamel demineralization. Caries Res 43: 278-285.

24. Mccullagh C, Robertson J, Bahnemann DW, Robertson P (2007) The Application of TiO2 Photocatalysis for Disinfection of Water Contaminated with Pathogenic Micro-Organisms: A Review Res Chem Intermed 33: 359-375.

25. Kim GC, Kim GJ, Park SR, Jeon SM, Seo HJ, et al. (2009) Air plasma coupled with antibody-conjugated nanoparticles: a new weapon against cancer. J Phys D: Appl Phys 42: 032005

26. Louroussi M (2005) Low Temperature Plasma-Based Sterilization: Overview and State-of-the-Art. Plasma Process Polym 2: 391-400.

27. Whittaker AG, Graham EM, Baxter RL, Jones AC, Richardson PR, et al. (2004) Plasma cleaning of dental instruments. J Hosp Infect 56: 37-41.

28. YangHong L, Liu S, Hu T (2013) Application of low-temperature plasma in dental clinical sterilization. Foreign Med Sci Stomatol 40: 483-485.

29. Sung SJ, Huh JB, Yun MJ, Myung B, Chang W, et al. (2013) Sterilization effect of atmospheric pressure non-thermal air plasma on dental instruments. J Adv Prosthodont 5: 2-8.

30. Sladek REJ, Stoffels E, Walraven R, Tiebeek PJA, Koolhoven RA(2004) Plasma treatment of dental cavities: a feasibility study. IEEE Trans Plasma Sci 32: 15401543.

31. Goree J, Liu B, Drake D, Stoffels E (2006) Killing of S. mutans Bacteria Using a Plasma Needle at Atmospheric Pressure. IEEE Trans Plasma Sci 34: 1317 1324.

32. Yang B, Chen J, Yu Q, Li H, Lin M, et al. (2011) Oral bacterial deactivation using a low-temperature atmospheric argon plasma brush. J Dent 9: 48-56.

33. Rupf S, Idlibi AN, Marrawi FA, Hannig M, Schubert A, et al. (2011) Removing biofilms from microstructured titanium ex vivo: a novel approach using atmospheric plasma technology. PLoSOne 6: e25893.

34. Koban I, Holtfreter B, Hübner NO, Matthes R, Sietmann R, et al. (2011) Antimicrobial efficacy of non-thermal plasma in comparison to chlorhexidine against dental biofilms on titanium discs in vitro - proof of principle experiment. J Clin Periodontol 38: 956-965.

35. Jiang CQ, Chen MT, Gorur A, Schaudinn C, Jaramillo DE, et al. (2009) Nanosecond pulsed plasma dental probe. Plasma Processes Polym 6: 479483.

36. Schaudinn C, Jaramillo D, Freire MO, Sedghizadeh PP, Nguyen A, et al. (2013) Evaluation of a non thermal plasma needle to eliminate ex vivo biofilms in root canals of extracted human teeth. Int Endod J 46: 1-8.

37. Koban I, Matthes R, Hübner NO, Welk A, Meisel P, et al. (2010) Treatment of Candida albicans biofilms with low-temperature plasma induced by dielectric barrier discharge and atmospheric pressure plasma jet. New J Phys 12: 073039.

38. Yamazaki H, Ohshima T, Tsubota $\mathrm{Y}$, Yamaguchi $\mathrm{H}$, Jayawardena JA, et al. (2011) Microbicidal activities of low frequency atmospheric pressure plasma jets on oral pathogens. Dent Mater J 30: 384-391. 
39. Lu X, Cao Y, Yang P, Xiong Q, Xiong Z, et al. (2009) An RC Plasma Device for Sterilization of Root Canal of Teeth. IEEE Trans Plasma Sci 37: 668-673.

40. Pan J, Sun K, Liang Y, Sun P, Yang X, et al. (2013) Cold plasma therapy of a tooth root canal infected with enterococcus faecalis biofilms in vitro. $J$ Endod 39: $105-110$

41. Rupf S, Lehmann A, Hannig M, Schäfer B, Schubert A, et al. (2010) Killing of adherent oral microbes by a non-thermal atmospheric plasma jet. J Med Microbiol 59: 206-212.

42. Kong MG, Kroesen G, Morfill G, Nosenko T, Shimizu T, et al. (2011) Plasma medicine: an introductory review. New J Phys 11: 115012

43. Ritts AC, Li H, Yu Q, Xu C, Yao X, et al. (2010) Dentin surface treatment using a non-thermal argon plasma brush for interfacial bonding improvement in composite restoration. Eur J Oral Sci 118: 510-516.

44. Yavirach $P$, Chaijareenont $P$, Boonyawan $D$, Pattamapun $K$, Tunma $S$, et al. (2009) Effects of plasma treatment on the shear bond strength between fiber reinforced composite posts and resin composite for core build-up. Dent Mater J 28: 686-692.

45. Lee HW, Kim GJ, Kim JM, Park JK, Lee JK, et al. (2009) Tooth bleaching with non thermal atmospheric pressure plasma. J Endod 35: 587-591.

46. Lee HW, Nam SH, Mohamed AAH, Kim GC, Lee JK (2010) Atmospheric Pressure Plasma Jet Composed of Three Electrodes: Application to Tooth Bleaching. Plasma Process Polym 7: 274-280.

47. Sun P, Pan J, Tien Y, Bai N, Wu H, et al. (2010) Tooth Whitening With Hydrogen Peroxide Assisted by a Direct-Current Cold Atmospheric-Pressure Air Plasma Microjet. IEEE Trans Plasma Sci 38: 1892-1896.
48. Park JK, Nam SH, Kwon HC, Mohamed AAH, Lee JK, et al. (2011) Feasibility of non thermal atmospheric pressure plasma for intracoronal bleaching. Int Endod J 44: $170-175$

49. Kim MS, Koo IG, Choi MY, Jung JC, Eldali F, et al. (2012) Correlated Electrical and Optical Studies of Hybrid Argon Gas-Water Plasmas and their Application to Tooth Whitening. Plasma Process Polym 8: 339-345.

50. Nam SH, Lee HW, SH Cho JKLEE, Jeon YC, Kim GC (2013) High-efficiency tooth bleaching using non thermal atmospheric pressure plasma with low concentration of hydrogen peroxide. J Appl Oral Sci 21: 265-270.

51. Claiborne D, McCombs G, Lemaster M, Akman MA, Laroussi M (2013) Lowtemperature atmospheric pressure plasma enhanced tooth whitening: the next-generation technology. Int J Dent Hygiene.

52. Jamali A, Evans PD (2013) Plasma treatment and bleaching to remove bluestain from lodgepole pine sapwood. Eur J Wood Prod 71: 675-677.

53. Diwan R, Debta FM, Deoghare A, Ghom S, Khandelwal A, et al. (2011) Plasma therapy: an overview. Journal of Indian Academy of Oral Medicine and Radiology 23: 120-123.

54. Vandamme M, et al. (2012) ROS implication in a new antitumor strategy based on non-thermal plasma. Int J Cancer 130: 2185-2194.

55. Volotskova O, Shashurin A, Stepp MA, Pal-Ghosh S, Keidar M (2011) Plasmacontrolled cell migration: localization of cold plasma-cell interaction region. Plasma Medicine 1: 85-92.

56. Lippens S, Denecker G, Ovaere P, Vandenabeele P, Declercq W (2005) Death penalty for keratinocytes: apoptosis versus cornification. Cell Death and Differentiation 12: 1497-1508. 